wässerigen Opiansăurelösung aufrecht erhielt. Ich setzte während des fortwährenden Rtihrens der Mischung zanăchst $10 \mathrm{ccm} \mathrm{N.-Kalilauge,}$ dann nach einiger Zeit der Einwirkung $10 \mathrm{ccm}$ N.-Salzsäure hinzu. Erst jetzt ließ.ich die Mischung sich trennen und schied die beiden Schichten von einander. Die Chloroformlösung hinterließ nach dem Verdunsten eine geringe Menge eines weißgelblichen Körpers, der bei $145^{\circ}$ schmolz und sich dadurch als unveränderte Opiansäure kennzeichnete. Aus der wässerigen Lösung schieden sich nach kurzer Zeit weiße Nadeln ab, die sich ebenfalls durch ihren Schmelzpunkt $=145^{\circ}$ als Opiansäure erwiesen.

Derselbe Versuch in derselben Anordnung, nur bei einer Temperatur von $60^{\circ}$ gehalten, wiederholt, ergab dasselbe negative Resultat.

\title{
4e. Ueber das Tarkoninmethyljodid und seine Beziehungen zu Cotarnin und Hydrocotarnin.
}

\author{
Von Dr. D. Bruns.
}

Nachstehende Experimental-Untersuchung tiber das Tarkoninmethyljodid wurde ausgetthrt, um an diesem verhältnismäßig leicht zugänglichen Material, das in seiner Konstitntion an das Corybalbin and seine Derivate erinnert, die einschlăgigen Verhältnisse zu stadieren und die Methoden kennen zu lernen, die anch bei dem schwerer zugănglichen Corybulbin und Isocorybulbin zam Ziele führen könnten. Sie bedentet daher eine Vorstadie für die bereits im Jahre 1903 in diesem Archiv niedergelegte Arbeit „Ueber Corybulbin und Isocorybulbin" "). Aber auch für unsere Kenntnisse über die Pseudoammoniumbasen ist diese Studie nicht ohne Bedeutung, denn sie zeigt uns, daß das Tarkoninmethyljodid, welches sich von dem Cotarninjodid nur durch einen Mindergehalt von zwei Wasserstoffatomen unterscheidet, also eine doppelte Bindung mehr hat, kaum noch die Fähigkeit besitzt, als ein Aldehyd zu reagieren. Es findet daher die von J. Gadamer in seiner Abhandlung uber die Konstitution der Pseudoammoniumbasen ansgesprochene Ansicht, $\mathrm{da} \subseteq$ die Beständigkeit der möglichen tautomeren Formen bei den einzelnen Basen sehr verschieden sein könne, volle Bestätigung. Wenn das Tarkoninmethylhydroxyd vielleicht noch als Aldehydbase auftreten kann, so ist dies sicher in viel geringerem

1) Band 241, 634 [1903]. 
Grade der-Fall als beim Berberin ${ }^{1}$ ), ja überhaupt scheint die Neigung zur Bildung der Pseudobasenform verhältnismäßig gering zu sein.

Noch von einem anderen Standpunkte aus war die Studie von Interesse. Sie lehrte nämlich die chromophore Gruppe im Berberin und den verwandten gelbgefärbten Verbindungen erkennen, oder bestätigt doch die auf andere Weise bereits gezogenen Schlüsse.

Bekanntlich geht das intensiv gelbe Berberin bei der Reduktion in das farblose Hydroberberin über, umgekehrt werden Cadadin (farblos) Corydalin, und Corybulbin durch Oxydation in die gelb gefärbten Verlindungen Berberin, Dehydrocorydalin und Dehydrocorybulbin verwandelt. Da hierbei $z$ wei Doppelbindungen zwischen $C$ und $C$ und zwischen $\mathrm{C}$ und $\mathrm{N}$ entstehen, mußten diese oder eine derselben die chromophoren Gruppen seiv. Das dann von J. Gadamer dargestellte Dihydroberberin, welches nur noch die Doppelbindung zwischen $\mathrm{C}$ und $\mathrm{C}$ aufweist, ist noch intensiv gelb gefärbt. Daraus konnte geschlossen werden, daß letztere Gruppe der Chromophor ist, wie aus nachstehender Zusammenstellang ersichtlich ist.

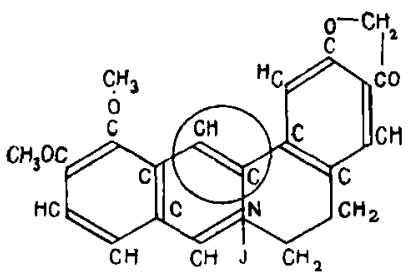

Berberinjodid gelb.

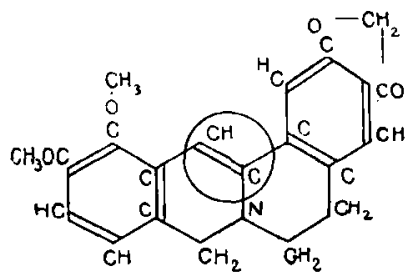

Dibydroberberin gelb.

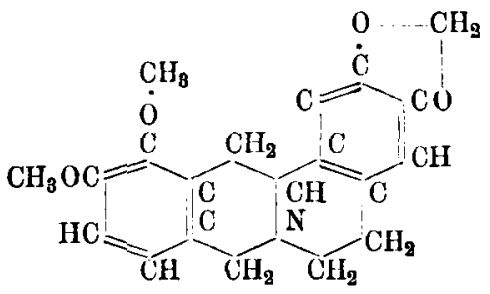

Tetrahydroberberin (Canadin)

farblos.

Dabei ist die chromophore Gruppe durch einen Kreis umschrieben.

1) Anm. Allerdings ist das Verhalten des Tarkoninnetbylhydroxyds gegen p-Dimethylamidoanilin noch nicht untersucht. Es wird dies jedoch jetzt nachgeholt werden. Die obige Arbeit von D. Bruns wurde bereits in Marburg ausgeführt und ist ein Teil seiner Dissertation. J. Gadamer. 
In einer ähnlichen Beziehung zu einander stehen Tarkoninmethyliodid, Cotarninjodid und Hydrocotarninjodid, indem der Wasserstoffgehalt von Verbindung zu Verbindung immer um zwel Atome zunimmt. Das Cotarninjodid ist also ein Dihydrotarkoninmethyljodid, das Hydrocotarnin ein Tetrahydroderivat des Tarkoninmethyljodids. Jedoch besteht ein Unterschied insofern, als das Cotarnin in seinen Salzen noch die Doppelbindung zwischen $\mathrm{C}$ and $\mathrm{N}$, nicht aber zwischen $\mathrm{C}$ und $\mathrm{C}$ anfweist. Ist also letztere Gruppe die chromophore, so maB Cotarnin tarblos sein, was ja in der Tat auch der Fall ist. Die Beziehungen dieser Verbindungen za einander illustriert die nachstehende Zusammenstellung:

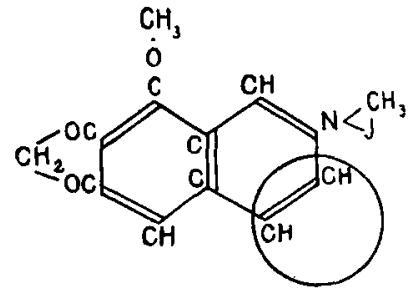

Tarkoninmethyljodid

gelb.<smiles>COc1cc(C(C)C)c2c(OC)c1OCC2</smiles>

\section{Experimenteller Teil.}

Tarkoninmethyljodid.

Das Tarkoninmethyljodid stellte ich mir durch Oxydation des Narkotins mit Jod in alkoholischer Lösung nach der Vorschrift Roser's her: ${ }^{1}$ )

Zu dem Zwecke löste ich $50 \mathrm{~g}$ Narkotin in $1000 \mathrm{~g} 80 \%$ igem Alkohol in der Wärme auf und gab zu der Lösung in längeren $Z$ wischenräumen $95 \mathrm{~g}$ Jod in drei Portionen $(60,20,15 \mathrm{~g})$ hinzu. Die Mischung warde dann 10 Stunden lang auf dem Dampfbade am RückfluBkühler erhitzt. Die nach

1) Ann. d. Chem. 245, 316 . 
dem Erkalten ausgeschiedenen Superjodide wurden abgesogen ${ }^{1}$ ), mit Wasser wiederum angerieben and unter zeitweiligem Erwărmen so lange Schwefelwasserstoff eingeleitet, bis die Braunfärbung völlig verschwanden und die Mischung rein gelb gefărbt war. Durch Auf kochen wurden die Jodide in Lösung gebracht, und die Lősung ron dem abgeschiedenen Schrefel heiB abfiltriert. Später zog ich es vor, die Superjodide durch Einleiten von Schwefligsäureanhydrid zu zerlegen. Dies Verfahren bot den Vorteil, daB die Reaktion rascher von statten ging, und die Abscheidung von Schwefel vermieden wurde. Die nach dem Erkalten ausgeschiedenen Krystalle bestehen aus einem Gemenge von Tarkoninmethyljodid und Jodtarkoninmethyljodid. Da letzterer Korrper bedeutend schwerer löslich in Wasser ist, wie das Tarkoninmethyljodid, so trennte ich die beiden Körper in der Weise, daß ich die Krystalle samt der Mutterlauge langsam erwärmte. Bei einer bestimmten Temperatur zerfallen die Krystalle, ein Teil geht in Lösung, während ein anderer Teil als schweres, etwas dunkler gelbes Pulver zu Boden fält. Von letzterem, dem Jodtarkoninmetbyljodid filtrierte ich die Lösung möglichst rasch ab und wiederholte mit den aus dieser nach dem Erkalten abgeschiedenen Krystallen das Verfahren noch einmal.

Ich erhielt so das Tarkoninmethyljodid in drusenförmig angeordneten gelben Nadeln, die bei $192^{\circ}$ scharf schmolzen. Die Ausbeute aus $50 \mathrm{~g}$ Narkotin betrng $20 \mathrm{~g}$ Tarkoninmethyljodid.

$0,2887 \mathrm{~g}$ gaben, bei $100^{\circ}$ getrocknet, nichts ab.

0,2887 g gaben $0,1930 \mathrm{~g} \mathrm{Ag} \mathrm{J}$.

Berechnet für $\mathrm{C}_{12} \mathrm{H}_{12} \mathrm{NO}_{3} . \mathrm{J}$ : J 36,8

Gefunden: 36,2 .

\section{Cotarnin.}

Das flir meine weiteren Versuche notwendige Cotarnin stellte ich mir aus dem Narkotin durch Oxydation mit verdünnter Salpetersăure her ${ }^{2}$ ).

$40 \mathrm{~g}$ Narkotin wurden mit $112 \mathrm{~g}$ Salpetersăure vom spez. Gew. 1,4 und $320 \mathrm{~g}$ Wasser unter ständigem Umrühren längere Zeit bei einer konstanten Temperatur von $49^{\circ}$ gehalten. Das Narkotin schmilzt zanăchst und löst sich dann auf. Bald nach erfolgter Lösung beginnen sich in der Flüssigkeit Flocken, die von Teropiammon herrühren, abzuscheiden. Sobald diese sich nicht mehr vermehren, läßt man erkalten, filtriert von den Flocken $a b$ und fällt das gebildete Cotarnin durch überschüssige Kalilauge aus. Nach dem Absaugen krystallisiert man es aus Benzol um.

Das Cotarnin bildet farblose Nadeln vom Schmp. $132^{\circ}$.

1) Aus den Mutterlaugen wurde noch das andere Spaltangsprodukt, die Opiansäure, gewonnen. (Siehe dort.)

2) Andersen, Ann. d. Chem. 86, 187. 
Das Tarkoninmethyljodid sowohl, wie das Cotarnin versuchte ich durch Behandlung mit Zink und verdünnter Schwefelsäure in die Tetrahydroverbindung, das Bydrocotarnin tiberzuführen. Whihrend das Tarkoninmethyljodid anf diesem Wege noch nicht reduziert worden ist, ist aus dem Cotarnin durch Einwirkung von Zink und Salzsäure in der Kälte ${ }^{1}$ ) bereits das Hydrocotarnin gewonnen worden. Mir gelang es in beiden Fallen, auf diesem Wege die gewunschte Verbindung za erhalten.

\section{Hydrocotarnin aus Tarkoninmethyljodid.}

$5 \mathrm{~g}$ Tarkoninmethyljodid löste ich in Wasser aut, setzte angeätztes granuliertes Zink und verdünnte Schwefelsăare zu und erwärmte die Mischung so lange auf dem Damptbade, bis die vorher intensiv gelb gefärbte Flüssigkeit farblos geworden war. Nach dem Erkalten filtrierte ich die Lösung in einen Scheidetrichter, machte sie mit $30 \%$ Ammoniak alkalisch und schittelte sie mit Aether mehrmals aus. Die filtrierten ätherischen Lðsungen wurden bis auf einen kleinen Rest abdestilliert, und der Rest in einer Krystallisierschale der freiwilligen Verdunstung uberlassen. Es hinterblieben tarblose, zu Rosetten angeordnete Krystalle, die den dem Hydrocotarnin zukommenden Schmelzpunkt $\left.{ }^{2}\right)=5 B^{0}$ zeigten.

Hydrocotarninbromid.

Zur weiteren Kennzeichnung des gewonnenen Hydrocotarnins führte ich dasselbe in das Bromsalz uber. Zn dem Zwecke loste ich $1 \mathrm{~g}$ Hydrocotarnin in Alkohol and versetzte die Lösung bis zor schwach sauren Reaktion mit verdünter Bromwasserstoffsaure. Schon nach kurzer Zeit batten sich reichliche Mengen gut ausgebildeter, farb. loser Nadeln ausgeschieden, die ich noch einmal aus Wasser umkrystallisierte. Entgegen den Angaben der Literatur ${ }^{8}$ ) erwiesen sich die Krystalle als wasserfrei.

$$
\begin{aligned}
& 0,3030 \mathrm{~g} \text { gaben } 0,1896 \mathrm{~g} \mathrm{Ag} \mathrm{Br} \text {. } \\
& 0,3757 \text { " } 0,2351 \text { n } \\
& \text { Berechnet für } \\
& \mathrm{C}_{29} \mathrm{H}_{15} \mathrm{NO}_{8} \text {. } \mathrm{HBr} \text { : } \\
& \mathrm{Br} 26,5
\end{aligned}
$$

1) Beckett und Wright, Journ. of chem. soc. 28, 577.

2) Roser, Ann. d. Chem. 249, 171.

-) R oscoe-S chorl emmer, Organ. Chem. 8, VI, S. 322. 


\section{Hydrocotarnin aus Cotarnin.}

Die Reduzierang des Cotarnins flihrte ich in derselben Weise aus, wie die des Tarkoninmethyljodids.

$5 \mathrm{~g}$ Cotarnin wurden in mit Schwefelsăure angesănertem Wasser gelöst und granuliertes Zink hinzugegeben. Nach mehrstïndigem Erhitzen anf dem Dampfbade filtrierte ich die Lösung in einen Scheidetrichter, machte sie nach völligem Erkalten mit 30\% Ammoniak stark alkalisch und schüttelte sie dann mehrere Male mit Aether aus. Die filtrierten, atherischen Lösungen destillierte ich bis auf einen kleinen Rest $a b$ und überließ diesen dann der freiwilligen Verdunstung. Ich erhielt auch hier Nadeln vom Schmp. $55^{\circ}$, die ich zur weiteren Kennzeichnung in das Bromsalz verwandelte.

\section{Hydrocotarninbromid.}

Das Salz wurde in derselben Weise, wie vorher erörtert, dargestellt.

Die Krystalle erwiesen sich beim Trocknen bei $100^{\circ}$ auch hier als wasserfrei:

$0,2207 \mathrm{~g}$ gaben $0,1382 \mathrm{~g} \mathrm{Ag} \mathrm{Br}$.

Berechnet für $\mathrm{C}_{12} \mathrm{H}_{16} \mathrm{NO}_{3}$. $\mathrm{HBr}$ :

Br 26,5

Gefunden:

26,6 .

Nachdem ich auf diese Weise nachgewiesen hatte, daß das Tarkoninmethyljodid sowohl, wie das Cotarnin durch naszierenden Wasserstoff in saurer Lösung in derselben Weise zu Hydrocotarnin reduziert wird, wie das Dehydrocorydalin zum i.-Corydalin, versuchte ich nun auch umgekehrt die Oxydation des Hydrocotarnins zum Tarkoninmethyljodid mit Jod in alkoholischer Lösung zu bewerkstelligen, eine Reaktion, die der von Ziegenbein bewirkten Oxydation des naturellen Corydalins vollstăndig entsprechen würde. Da es mir nun daran lag, die Bedingungen festzustellen, unter denen das Zwischenprodukt, das Cotarnin, hier entstehen und isoliert werden könnte, versuchte ich weiter durch gemäßigte Oxydation mit Jod einerseits aus dem Hydrocotarnin das Cotarnin und weiter auch aus dem Cotarnin das Tarkoninmethyljodid herzustellen. Es ist mir gelungen, in allen Fällen die Bildung der gewïnschten Oxydationsstufen festzustellen, wenn auch der Prozeb nicht immer gleich glatt zu verlaufen scheint.

\section{Oxydation des Hydrocotarnins mit Jod.}

Der Versuch warde in derselben Weise angestellt, in der Ziegenbein die Oxydation des Corydalins gelungen war.

$0,1210 \mathrm{~g}$ zuvor iber Schwefelsäure getrocknetes Hydrocotarnin wurden in einer Druckflasche mit $50 \mathrm{ccm}$ einer empirisch hergestellten 
Jodlösung (2 $\mathrm{g}$ Jod in Alkohol gelost, auf $100 \mathrm{ccm}$ aufgef üllt) übergossen und drei Stunden lang im Autoklaven auf 95-100 erhitzt. Nach dem Erkalten lagen die gebildeten Perjodide als dunkelbraunrote, formlose Massen unten am Boden der Flasche. Ich setzte nun 2 g Jodkalium, etwas $\mathrm{NaHCO}_{8}$, um die entstandene Jod wasserstoffsăore zu neutralisieren, und $100 \mathrm{ccm} \mathrm{n/10} \mathrm{Natriumthiosulfatlossung} \mathrm{hinzu.} \mathrm{Die}$ Flüssigkeit färbte sich sofort schwach gelblich. Um anch die letzten Reste der Perjodide zu zerlegen, schüttelte ich die Mischung im Schlittelapparat so lange, bis alles gelöst war. Ich füllte sodann im Maßkolben aut $500 \mathrm{ccm}$ anf und verwendete jedesmal $100 \mathrm{ccm}$ der Lösung, um die überschüssig zugesetzte $\mathrm{n} / 10$ Natriumthiosulfatlosung mit $\mathrm{n} / 10$ Jodlösung - Stärkelösung als Indikator - zurückzutitrieren. Der Farbenumschlag war gut za erkennen. Die Berechnung ergab, daß $0,1210 \mathrm{~g}$ Hydrocotarnin zur Oxydation $0,273 \mathrm{~g}$ Jod verbrancht hatten. Nach der Gleichung

$$
\mathrm{C}_{12} \mathrm{H}_{13} \mathrm{NO}_{8}+4 \mathrm{~J}=\mathrm{C}_{22} \mathrm{H}_{12} \mathrm{NO}_{8} \mathrm{~J}+3 \mathrm{HJ}
$$

berechnen sich $0,277 \mathrm{~g}$. Die Reaktion war also anscheinend glatt verlaufen.

Wie Gadamer ${ }^{1}$ ) nachgewiesen hat, geht die quantitative Oxydation mit Jod in alkobolischer Lösung nicht immer in so einfacher Weise, wie oben, vor sich, da einerseits das Jod auch zersetzend auf den Alkohol einwirkt, andererseits die Oxydationswirkung auf die Base durch die Bildung jodwasserstoffsaurer Salze verlangsamt wird. Immerhin ist durch die Erfahrung bei der Oxydation des Corydalins, Tetrahydroberberins u. s. w. bewiesen worden, daß, wenn die Base überhaupt oxydierbar ist, die Methode praktisch genügend zuverlässige Resultate liefert. Wir konnen also aus dem Ergebnis unseres Versuches mit Bestimmtheit den Schluß ziehen, daß das Hydrocotarnin durch das Jod bis zum Tarkoninmethyljodid oxydiert worden ist.

\section{Oxydation des Hydrocotarnins zu Cotarnin.}

Ich wollte nan ferner versuchen, ob ich durch Anwendung einer für obige vollstăndige Oxydation ungenügenden Menge Jod vielleicht zum Cotarnin, dem um zwei Wasserstoffatome årmeren Körper, gelangen könnte. Um nun aber, falls die quantitative Oxydation nicht so ganz glatt verlaufen sollte, die Isolierung der gebildeten Produkte zu ermöglichen, verwandte ich flir diesen Versuch eine größere Menge Hydrocotarnin.

$0,9104 \mathrm{~g}$ Hydrocotarnin wurden mit $75 \mathrm{ccm}$ alkoholischer Jodlösung (3 $\mathrm{g} \mathrm{J}$ auf $100 \mathrm{ccm}$ ) 9 Stunden lang im Autoklaven erhitzt.

1) Arch. d. Pbarm. 1902, S. 32. 
Nach dem Erkalten waren große Mengen lichtbrauner Nadeln auskrystallisiert, während die obenstehende Flüssigkeit nur mehr bellrot gefärbt war. Ich setzte nun wieder $2 \mathrm{~g}$ Jodkalium, etwas Natriumbikarbonat und $150 \mathrm{ccm} 1 / 10 \mathrm{Na}_{2} \mathrm{~S}_{2} \mathrm{O}_{\mathrm{a}}$-Lösung hinzu, schtittelte bis zar völligen Lösung und füllte dann auf $500 \mathrm{ccm}$ anf und titrierte in einem aliquoten Teil den Ueberschuß an n/10 Natriumthiosulfatlösang zarück.

Es ergab sich, daß zur Oxydation $0,7747 \mathrm{~g}$.Jod verbraucht waren, während nach der Gleichung

$$
\mathrm{C}_{18} \mathrm{H}_{15} \mathrm{NO}_{8}+2 \mathrm{~J}=\mathrm{C}_{18} \mathrm{H}_{16} \mathrm{NO}_{8} \mathrm{~J}+\mathrm{HJ}
$$

$1,04 \mathrm{~g}$ berechnet sind.

Die Reaktion scheint nach diesem Befunde nur unvollständig verlaufen zu sein, und ich versuchte daher, um mit Sicherheit über die etwaige Bildung von Cotarnin entscheiden zu können, das Oxydationsprodukt selbst zu isolieren. Zu dem Zwecke dunstete ich den Rest der mit überschüssigem Natriumthiosulfat versetzten Lösung auf dem Dampfbade vorsichtig zur Trockne ein und zog den Trockenrückstand mehrmals mit Alkohol aus. Nach dem Verdunsten der filtrierten alkoholischen Lösung hinterblieben nadelförmige Krystalle, die ich wiederum in Wasser loste. Die Lösung machte ich sodann mit Kalilauge alkalisch und schlittelte sie mit Aether aus. Die ätherische Lösung hinterließ dann nach dem freiwilligen Verdunsten gelbliche Nadeln, die durch ihren Schmp. $=130^{\circ}$ als Cotarnin gekennzeichnet wurden.

Es war also tatsächlich bei dieser gemäßigten Oxydation aus dem Hydrocotarnin Cotarnin gebildet worden, wenn die Reaktion auch nicht ganz glatt verlaufen ist.

\section{Oxydation des Cotarnins zu Tarkoninmethyljodid.}

Um auch das zweite Glied der Oxydation, die Ueberf tuhrung des Cotarnins in Tarkoninmethyljodid, zur Durchführung zu bringen, ubergo $B$ ich in einer Druckflasche $0,7432 \mathrm{~g}$ Cotarnin mit $75 \mathrm{ccm}$ einer empirischen Jodlösung ( $3 \mathrm{~g}$ Jod auf $100 \mathrm{ccm}$ ) und erhitzte 12 Stunden lang im Autoklaven auf $95-100^{\circ}$. Nach dem Erkalten hatten sich die Superjodide in Form dunkelbrauner, feiner Nadeln abgeschieden. Ich setzte wiederum $2 \mathrm{~g}$ Jodkalium, etwas $\mathrm{NaHCO}_{8}$ und $200 \mathrm{ccm}$ $1 / 10 \mathrm{Na}_{2} \mathrm{~S}_{2} \mathrm{O}_{8}$-Lősung za, erwărmte bis zur Lősung der Perjodide und füllte nach dem Erkalten auf $500 \mathrm{ccm}$ ant. Die Rucktitration ergab einen Verbrauch von $1,09 \mathrm{~g}$ Jod, während nach der Formel

$$
\mathrm{C}_{12} \mathrm{H}_{15} \mathrm{NO}_{4}+2 \mathrm{~J}=\mathrm{C}_{28} \mathrm{H}_{12} \mathrm{NO}_{8} \mathrm{~J}=\mathrm{H}_{8} \mathrm{O}+\mathrm{HJ}
$$

nur $0,795 \mathrm{~g} \mathrm{Jod}$ berechnet sind. 
Die Differenzen sind also hier besonders groß. Es ist sehr wabrscheinlich, daß die Fehlerquellen, die Gadamer') bei dem quantitativen Oxydieren nit Jod in alkoholischer Lösung nachgewiesen hat, sich bei den größeren Mengen, die ich bei den letzten Versuchen von der Base sowohl, wie vom Jod anwandte, erheblich verstärkten.

Auch hier versuchte ich deswegen, um die Frage nach dem Oxydationsprodukt entscheiden zu können, letzteres aus der Lösung zu isolieren. Zu dem Zweck dampfte ich den Rest der nicht zur Rücktitration verwandten Flüssigkeit vorsichtig auf dem Dampfbade zur Trockne ein und zog den Trockenrïckstand mit Alkohol aus. Die filtrierte alkoholische Lösung ließ ich freiwillig verdunsten und krystallisierte den Räckstand noch einmal aus Wasser um. Ich erhielt so kleine, gelbe Nadeln, die den Schmp. $18 \bar{\imath}-189^{\circ}$ zeigten. Da der Schmelzpunkt des reinen Tarkoninmethyljodids bei $192^{\circ}$, der des Cotarninjodids bei $165^{\circ}$ liegt, so dürfte durch den obigen Schmelzpunkt bewiesen werden, daß die erhaltenen gelben Nadeln wirklich Tarkoninmethyljodid waren, daß also das angewandte Cotarnin durch die Oxydation mit Jod wenigstens teilweise in Tarkoninmethyljodid überget ührt worden ist.

Bei der Behandlung mit überschüssigem Jod stellt sich das Verhalten des Hydrocotarnins also völlig dem des Tetrahydroberberins und des Corydalins an die Seite. Auch hier wird glatt ein um vier Wasserstoffatome ärmeres Dehydroderivat gebildet. Durch die beiden letzten Versuche warde anch bewiesen, daB die Oxydation des Hydrocotarnins zum Tarkoninmethyljodid tatsächlich uber das Cotarnin hinweg. führte. Jedoch scheinen sich diese graduell verschiedenen Oxydationen alle mehr oder weniger nebeneinander zu vollziehen, so daß man nur dann hoffen konnte auch aus dem Corybulbin den um zwei Wasserstoffatome ärmeren Korper isolieren zu können, wenn man genligend große Mengen des Ausgangsmaterials zu dem dahinzielenden Versuche verwenden konnte.

Wahrend ich so die vollige Uebereinstimmung des Tarkoninmethyljodids mit Berberin und Dehydrocorydalin und ibrer Tetrahydroverbindungen in ihrem Verhalten gegen naszierenden Wasserstoff und Jod, wie erwartet, feststellen konnte, gestalteten sich die Verhältnisse ungtiostiger, als ich das Tarkoninmethyljodid mit Aceton, Chloroform and Schwefelammonium in Wechselwirkung zu bringen versuchte und weiter auch sein Verhalten gegen starke Kalilauge und Hydroxylamin einer Prüfung unterzog. Alle diese Versuche scheiterten. Es wurde allerdings durch die Einwirkung von $30 \%$ Kalilauge zunăchst ein

1) a. a. 0 .

Arch. d. Pharm. UCXXXXIII. Bds. 1. Heft. 
Körper gebildet, der mit alkalischer Reaktion beim Ausschütteln mit Aether in diesen hineinging, aber sehr bald trat unter Auftreten gelber bis roter Färbungen eine weitergehende Zersetzung ein. Doch sollen die ausgeführten Versuche in folgendem kurz beschrieben werden.

\section{Einwirkung von Acoton auf Tarkoninmethyljodid.}

0,5 $\mathrm{g}$ Tarkoninmethyljodid kochte ich mit $5 \mathrm{ccm}$ Aceton am Rück flaßkühler, bis Lösung eingetreten war, und machte diese dann mit $30 \%$ Natronlauge stark alkalisch. Es schied sich hierbei ein sehr dunkel gefärbter harziger Niederschlag aus, der auch nach längerem Stehen in der Kälte nicht fest werden wollte. Nach monatelangem Aufbewahren im Exsiccator begann er an einigen Stellen fest zu werden. Vielleicht lag hier die Acetonverbindung vor, doch war der Körper in der mir vorliegenden Form nicht analysierbar.

Bei einem zweiten Versuch löste ich $0,5 \mathrm{~g}$ Tarkoninmethyljodid in möglichst wenig Wasser, fügte etwas Aceton hinzu und machte die Mischung mit gesättigtem Barytwasser stark alkalisch. Die Flüssigkeit blieb auch nach einigem Erwärmen völlig klar. Gut verschlossen, in der Kälte sich selbst überlassen, veränderte sich die Lösung nicht.

\section{Einwirkung von Chloroform auf Tarkoninmethyljodid.}

W'enn ich Tarkoninmethyljodid mit Chloroform zusammenbrachte, löste es sich darin auf, ohne auch nach dem Zusatz von starker Kalilauge eine Verbindung damit einzugehen. Ich versuchte nun so zum Ziele zu gelangen, daß ich das leicht lösliche Sulfat ${ }^{1}$ ) in möglichst wenig Wasser löste, mit Chloroform versetzte und mit $30 \%$ Kalilange stark alkalisch machte. Nach längerem kräftigen Umschütteln unter zeitweiligem Erwärmen zeigte sich das Chloroform rotgelb gefärbt. Nach freiwilligem Verdunsten hinterließ die Lơsung einen roten harzartigen Körper, der sich in Alkohol glatt löste - Berberin- und Dehydrocorydalinchloroform lösen sich nicht in Alkohol - und weder aus diesem, noch aus anderen Lösungsmitteln krystallinisch erhalten werden konnte. Also scheint auch hier die Reaktion nicht eingetreten zu sein.

\section{Einwirkung von Schwefelammonium auf Tarkoninmethyljodld.}

$0,5 \mathrm{~g}$ Tarkoninmethyljodid lőste ich in verdünntem Alkohol auf and versetzte die noch warme Lösung mit dem gleichen Volumen dunkelgelben Schwefelammoniums. Die Farbe der Lösung verănderte sich hierbei kaum. Nach längerem Stehen in der Kälte war ein

1) Darstellung siehe später. 
Niederschlag entstanden, der nach dem Absaugen und raschem Aus. waschen mit Alkohol und Aether weißlichgelb aussah. Durch Uebergießen mit verdünnten Säuren konnte keine Farbenänderung und kein Geruch nach Schwefelwasserstoff wahrgenommen werden, so daß sich ein Polysulfid nicht gebildet zu haben schien, oder - wegen Leichtlöslichkeit nicht zar Abscheidung gekommen war.

Um die Einwirkung starker Kalilauge auf das Methyltarkoninmhydroxyd untersuchen zu können, schien es mir angebracht, zunächst das schwefelsaure Salz der Báse herzustellen. Ich konnte dann aus dessen wässeriger Lösung durch die berechnete Menge titrierten Barytwassers die Schwefelsäure ansfällen and die so gewonnene Lősung der freien Base mit $30 \%$ Kalilange behandeln.

\section{Tarkoninmethylsulfat.}

5 g Tarkoninmethyljodid löste ich in Wasser auf und digerierte die Lösung einige Zeit mit uberschüssigem Silbersulfat. Ich filtrierte dann heiß vom ausgeschiedenen Jodsilber ab, wusch mehrere Male mit heißem Wasser nach and befreite die Lösung durch Einleiten von Schwefelwasserstoff vom gelosten Silbersulfat. Nachdem ich dann das ausgeschiedene Schwefelsilber durch Filtrieren beseitigt und den Schwefelwasserstoff durch Erwärmen verjagt hatte, dampfte ich die Lösung auf dem Wasserbade bis auf ein ein kleines Volumen ein. Da nichts auskrystallisierte, ließ ich die Lösung im Exsiccator trocken werden. Der Rückstand, eine gelbgraue Masse, wurde ans Alkohol umkrystallisiert.

So gewonnen, bildet das Tarkoninmethylsulfat lange, schwach gelbliche, in Drusen angeordnete Nadeln, die sich äußerst leicht in Wasser, leicht in Alkohol lösten. Die wässerige Lðsung fluoresziert grünlich.

$0,8183 \mathrm{~g}$ gaben $0,1560 \mathrm{~g} \mathrm{Ba} \mathrm{SO}$.

Berechnet für:

$\mathrm{C}_{19} \mathrm{H}_{18} \mathrm{NO}_{8}$. $\mathrm{HSO}_{4}: \quad\left(\mathrm{C}_{22} \mathrm{H}_{19} \mathrm{NO}_{3}\right)_{2} \mathrm{SO}_{4}$ :

$\mathrm{SO}_{4} 30,5 \quad 29,5$

Es lag hier mithin ein saures Salz vor.

\section{Einwirkung starker Kalilauge auf Methyltarkoniumhydroxyd.}

$3 \mathrm{~g}$ Tarkoninmethylsulfat löste ich in Wasser und fällte durch den Zusatz einer berechneten Menge titrierten Barytwassers die Schwefelsä̆ure aus. Ich filtrierte vom ausgeschiedenen Baryumsulfat heiß ab und versetzte die Lösung im Scheidetrichter mit $50 \%$ Natronlauge. Die Lösung trüte sich stark, ohne aber Flocken abzuscheiden. 
Ich schüttelte sodann die Lösung mit Aether ans, wobei die Trübung wieder verschwand. Die sofort abfiltrierte ätherische Iösung war zunäcbst farblos und klar; sie reagierte alkalisch. Nach kurzer Zeit bereits nahm sie aber eine gelbliche Färbung an, die allmählich immer mehr in Rot überging. Die Hälfte der ätherischen Lösung wurde zonächst auf ein kleines Volumen abdestilliert und der Rest in einer Schale verdunstet, während ich die andere Hälfte von vornherein der freiwilligen Verdunstung überließ. In beiden Fällen resultierten rote, ölige Rückstände, die auch nach längerem Stehen keine Neigung zur Krystallisation zeigten. Beim Uebergießen mit verdünnter Schwefelsåure trat keine Aufhellung und Losung ein, so daß an einer eingetretenen, weitergehenden Zersetzung wohl nicht zu zweifeln ist.

\section{Einwirkung von Hydroxylamin auf Methyltarkoniumhydroxyd.}

$\mathrm{Zu}$ diesem Versuche benutzte ich zunächst dieselbe Methode, die Gadamer') mit Erfolg angewendet hat, um das Berberinaloxim darzustellen. Zunächst hatte ich mir dazu eine alkoholisch-ätherische Lösung von freiem Hydroxylamin herzustellen.

Zn dem Zwecke rieb ich 2,2 g Hydroxylaminchlorhydrat mit $4,6 \mathrm{~g}$ krystallisiertem Natriumkarbonat in einer Porzellanschale zusammen und gab zu der hierdurch halbflussig gewordenen Masse $20 \mathrm{ccm}$ absolnten Alkohol. Um das freigewordene Kohlensäureanhydrid zu verjagen, erwärmte ich die Mischung unter Ersatz des verdampfenden Alkohols gelinde auf dem Dampfbade und spülte sie endlich mit Aether in einen $110 \mathrm{ccm}-K o l b e n$ hinein. Nach dem Erkalten füllte ich sodann mit Aether bis zur Marke auf und filtrierte nach dem Absetzen von dem ansgeschiedenen Chlornatrium durch ein trockenes Filter ab. Von der Lösung entsprach $1 \mathrm{ccm}=0,2 \mathrm{~g}$ salzsaurem Hydroxylamin.

Zur Ausführung des Versuches löste ich $1 \mathrm{~g}$ Tarkoninmethylsulfat in $5 \mathrm{ccm}$ Wasser, versetzte die Lösung mit $20-30 \mathrm{ccm} 30 \%$ iger Natronlauge und schüttelte die Mischung mehrmals mit Aether aus. Die sofort abfiltrierten ätherischen Lösungen wurden vereinigt und mit $20 \mathrm{ccm}$ obiger Hydroxylaminlösung versetzt. Es trat sofort eine schwache, gelbliche Trübung ein, doch gelang es mir nicht trotz sorgfảltiger Abkühlung eine krystallinische Abscheidung zu erhalten. Vielmehr wurde bereits nach kurzer Zeit die Färbung der Lösung eine schmutzig rotliche, und es hinterblieb nach freiwilligem Verdunsten des Aethers ein rötlicher, öliger Rückstand, ähnlich dem, den ich bei der Einwirkung der konzentrierten Kalilauge allein erhalten hatte. Auch hier löste er sich nicht in verdünnter Schwefelsäare, so daß dieselbe Zersetzung, wie oben, eingetreten sein dürfte.

1) Siehe die vorstehende Arbeit über das Berberin. 
Weiter versuchte ich noch auf dieselbe Weise zum Ziele zu gelangen, wie Roser ${ }^{1}$ ) das Cotarnin oximierte. $Z \mathbf{a}$ dem $Z$ wecke löste ich $1,2 \mathrm{~g}$ Tarkoninmethylsulfat in $75 \mathrm{ccm}$ absoluten Alkohol and fallte nun zunăchst durch die berechnete Menge titrierten Barytwassers die Schwefelsăure aus. Ich filtrierte dann die Lősung von dem Baryumsulfat, das sich recht voluminös abgeschieden hatte, durch ein Faltenfilter in ein Kolbchen, das $0,5 \mathrm{~g}$ Hydroxylaminchlorhydrat enthielt. Ich erwärmte dann die Mischung zwei Stunden lang auf dem Dampfbade und ließ sie einige Tage in der Kälte stehen. Als keine krystallinische Abscheidung eintrat, dunstete ich die alkoholische Lösung bei gelinder Wärme zur Trockne, nahm den Trockenrückstand wieder mit Wasser anf, filtrierte und setzte der blanken Lösung Natriumkarbonatlosung bis zur stark alkalischen Reaktion zu, um auf diese Weise aus etwa gebildetem Oximchlorid das Oxim selbst in Freiheit zu setzen und zur Ausfallung zu bringen. Da auch hierbei die Flibssigkeit vollig blank blieb, dürfte eine Oximierung des Tarkonins auch bei diesem Versuch nicht eingetreten sein.

Mitteilungen aus dem pharmazeutisch-chemischen Institut der Universität Marburg.

\section{Ueber Ameisensäure und deren titrimetrische Bestimmung.}

Von E. Rupp.

(Eingegangen den 28. I. 1905.)

Nach dem M. Goldschmidt'schen Patentverfahren ${ }^{2}$ ) wird seit einiger Zeit in großer Menge Ameisensiiure für technische Zwecke durch Einwirkung von Koblenoxyd auf gepulvertes Aetznatron unter Druck gewonnen.

Wie die Untersuchung ergibt, zeichnet sich die aus dem so entstandenen Natriumformiat in Freiheit gesetzte Säure durch einen verbältnismäßig hohen Grad von Reinheit aus, so daß hieraus an Hand der Hilfsmittel des pharmazeutischen Laboratorinms leicht ein den Ansprüchen des Arzneibuches entsprechendes Praparat gewonnen

1) Ann. d. Chem. 352, 337.

2) Inhaberin: Nitritfabrik Köpenick bei Berlin. 